\title{
Rings with orthogonality relations
}

\section{G. Davis}

\begin{abstract}
The rings of this paper are assumed to have relations of orthogonality defined on them. Such relations are uniquely determined by complete boolean algebras of ideals. Using the Stone space of these boolean algebras, and following J. Dauns and K.H. Hofmann, a sheaf-theoretic representation is obtained for rings with orthogonality relations, and the rings of global sections of these sheaves are characterized. Baer rings, $f$-rings and commutative semi-prime rings have natural orthogonality relations and among these the Baer rings are isomorphic to their associated rings of global sections. A special type of ideal is singled out in commutative semi-prime rings and following $G$. Spirason and E. Strzelecki, in an unpublished note, a characterization of a class of such rings is obtained.
\end{abstract}

\section{Orthogonality relations}

DEFINITION 1.1. A relation $*$ on a ring $R$ is said to be an orthogonality relation if

(1) $x * y$ implies $y * x$;

(2) $0 * x$ for all $x \in R$;

(3) $x * x$ implies $x=0$;

(4) $x_{1} * y, x_{2} * y$ implies $\left(x_{1}-x_{2}\right) * y$;

(5) $x * y$ implies $(a x) * y,(x a) * y$ for all $a \in R$;

(6) if 1 is a multiplicative identity for $R$ then $x * 1$ implies 


$$
x=0 \text {. }
$$

Examples of rings with orthogonality relations are

(1) commutative semi-prime rings with $x * y$ if $x y=0$;

(2) f-rings with $x * y$ if $|x| \wedge|y|=0$.

DEFINITION 1.2. For $x \in R, A \subseteq R$ put $x^{*}=\{y \in R ; x * y\}$, $A^{*}=\bigcap_{a \in A} a^{*}, A^{* *}=\left(A^{*}\right)^{*}$. If $A=\{x\}$ is a singleton set then $A^{*}=\{x\}^{*}$ will be written simply as $x^{*}$, and similarly for $x^{* *}$. A subset of $R$ of the form $A^{*}, A \subseteq R$ is said to be a polar subset of $R$. The class of all polar subsets of $R$ is denoted by $B(R)$.

The following properties of polar subsets are easily established:

(1) every polar subset of $R$ is a two-sided ideal;

(2) $0^{*}=R, R^{*}=(0)$;

(3) $\bigcap_{\alpha} A_{\alpha}^{*}=\left(\bigcup_{\alpha} A_{\alpha}\right)^{*}$ for any class $\left\{A_{\alpha}\right\}$ of subsets $A_{\alpha} \subseteq R$;

(4) $A^{*} \cap A^{* *}=(0)$ for any $A \subseteq R$.

The orthogonality relation * is said to be regular if $x^{* *} \cap y^{* *}=(0)$ implies $x * y$.

Unless a statement to the contrary is made it will be assumed that all orthogonality relations discussed are regular.

1.3. $B(R)$, ordered by inclusion, is a complete boolean algebra.

Proof. Using Frink's axioms [2] for a boolean algebra it is enough to see that $B(R)$ is a complete lower semi-lattice such that for any $A^{*} \in B(R)$ there is an $\left(A^{\prime}\right) * \in B(R)$ satisfying $\left(A^{\prime}\right) * \cap B^{*}=(0)$ if and only if $A^{*} \cap B^{*}=B^{*}$. In fact $A^{\prime}=A^{*}$ suffices: if $A^{*} \cap B^{*}=B^{*}$ then $A^{* *} \cap B^{*}=A^{* *} \cap\left(A^{*} \cap B^{*}\right)=\left(A^{* *} \cap A^{*}\right) \cap B^{*}=(0) \cap B^{*}=(0)$. In general $B^{*} \supseteq(A \cup B)^{*}=A^{*} \cap B^{*}$. Suppose that $A^{* *} \cap B^{*}=(0)$ and take $x \in B^{*}$. If $x \notin A^{*}$ then for some $a \in A, x * a$ is false. Then $x^{* *} \cap a^{* *} \neq(0)$ since * is regular, but $x^{* *} \subseteq B^{*}, a^{* *} \subseteq A^{* *}$ and $B^{*} \cap A^{* *}=(0)$. Thus $x \in A^{*}$ so that $B^{*}=A^{*} \cap B^{*}$.

A boolecon structure $B$ for a ring $R$ is a class of (two-sided) ideals of $R$ such that: 
(1) B, ordered by inclusion, is a complete boolean algebra;

(2) the zero of $B$ is (0) and the unit of $B$ is $R$.

Thus, if * is an orthogonality relation on $R$ then the class $B(R)$ of polar subsets of $R$ is a boolean structure. On the other hand if $B$ is a boolean structure for $R$ define $x^{\circ 0}=\Pi\{B \in B: x \in B\}$ and $x^{\circ}=$ complement of $x^{\circ 0}$ in $B$, for $x \in R$. The relation $*$ defined by $x * y$ if $x \in y^{\circ}$ is then an orthogonality relation on $R$ with $B(R)=B$.

\section{Representations}

In this section a representation theorem for rings with orthogonality relation is described. This representation is in terms of rings of global sections of sheaves and since this method of representation has been given at length elsewhere (Dauns and Hofmann [1], Kist [4], Pierce [5]) only the pertinent definitions and proofs of the final results will be given here.

DEFINITION 2.1. If $R, R^{\prime}$ are rings with orthogonality relations denoted indifferently by $*$ then a map $h: R \rightarrow R^{\prime}$ is a $*$-isomorphism if

(1) $h$ is a ring isomorphism into $R^{\prime}$,

(2) $x * y$ if and only if $h(x) * h(y)$.

DEFINITION 2.2. A triple $(A, b, C)$ is a sheaf of rings if

(1) $A, C$ are topological spaces;

(2) $b: A+C$ is a local homeomorphism (that is for each $a \in A$ there is an open set containing $a$ such that $b$ restricted to this open set is a homeomorphism onto an open subset of $C$ );

(3) for each $\gamma \in C, b^{-1}(\gamma)$ is a ring in $A$;

(4) the maps $\left(\alpha_{1}, \alpha_{2}\right)+\alpha_{1}-\alpha_{2},\left(\alpha_{1}, \alpha_{2}\right)+\alpha_{1} \alpha_{2}$ from the set $A \vee A=\left\{\left(\alpha_{1}, \alpha_{2}\right) \in A \times A: b\left(\alpha_{1}\right)=b\left(\alpha_{2}\right)\right\}$ into $A$ are continuous.

If $C^{\prime} \subseteq C$ is an open set then a continuous map $\sigma: C^{\prime} \rightarrow A$ is said to be a section over $C^{\prime}$ if boo : $C^{\prime}+C^{\prime}$ is the identity map on $C^{\prime}$. If $C^{\prime}=C$ then a section over $C^{\prime}$ is said to be a global section. The set of all global sections is denoted by $\Gamma(A)$ and is a ring for the 
pointwise operations: $[\sigma+\tau](\alpha)=\sigma(\alpha)+\tau(\alpha), \sigma \cdot \tau(\alpha)=\sigma(\alpha) \cdot \tau(\alpha)$.

If $R$ is a ring with an orthogonality relation * write $Q$ for the Stone space of the boolean algebra $B(R)$ of polar subsets of $R$. Thus, $Q$ is a Hausdorff extremally-disconnected topological space.

DEFINITION 2.3. For $t \in Q$ define $R_{t}=\left\{x \in R: x^{*} \in t\right\}$.

LEMMA 2.4. Every $R_{t}, t \in Q$, is a (two-sided) ideal of $R$, $\bigcap_{t \in Q} R_{t}=(0)$. Every $R_{t}$ is a prime ideal if and only if $x * y$ is equivalent to $x y=0$.

Proof. It is straightforward to check that each $R_{t}$ is an ideal and $\bigcap_{t \in Q} R_{t}=(0)$. Suppose that $x * y$ is equivalent to $x y=0$. Thus $x y=0$ implies $x * y$ which in turn implies $x^{* *} \cap y^{* *}=(0)$ so that for each $t \in Q$ either $x^{* *} \in t$ or $y^{* *} \epsilon t$. That is for each $R_{t}$ either $x \in R_{t}$ or $y \in R_{t}$ so that each $R_{t}$ is prime. Conversely, suppose that each $R_{t}$ is prime. If $x y=0$ then $x \in R_{t}$ or $y \in R_{t}$, for each $t \in Q$ so that $x^{* *} \cap y^{* *} \in \prod_{t \in Q} t=(0)$ and thus $x * y$. In general if $x * y$ then $x y=0$, for in this case $(x y) * * \subseteq x^{* *} \cap y^{* *}=(0) . \quad / /$ DEFINITION 2.5. For $t \in Q$ write $R / R_{t}$ for the set of ordered pairs $\left(x+R_{t}, t\right), x \in R$. Then $R / R_{t}$ is a ring for the operations

$$
\begin{aligned}
\left(x+R_{t}, t\right)+\left(y+R_{t}, t\right) & =\left(x+y+R_{t}, t\right), \\
\left(x+R_{t}, t\right)\left(y+R_{t}, t\right) & =\left(x y+R_{t}, t\right) .
\end{aligned}
$$

Put $R=\bigcup_{t \in Q} R / R_{t}$ and define $p: R \rightarrow Q$ by $p(r)=t$ if $r \in R / R_{t}$. For each $x \in R$ define a map $\hat{x}: Q \rightarrow R$ by $\hat{x}(t)=(x+R, t)$. Put $\hat{R}=\{\hat{x}: x \in R\}$.

LEMMA 2.6. For each $x \in R, p \cdot \hat{x}$ is the identity mop on $Q$ and the set $\hat{R}$ is a ring for the pointwise operations.

A base for the open sets for the hull-kernel topology on $Q$ consists 
of sets of the form $Q_{A}=\left\{t \in Q: A^{*} \forall t\right\}$. The sets $\hat{x}\left(Q_{A}\right)=\left\{\hat{x}(t): t \in Q_{A}\right\}$ for $x \in R, A \subseteq R$, then form a base for the open sets for a topology on $R$.

PROPOSITION 2.7. With the hull-kemel topology on $Q$, and the topology described above on $R$, the triple $(R, p, Q)$ is a sheaf of mings.

Proof. Similar to Proposition 2.13 of Dauns and Hofmann [1].

The ring $\Gamma(R)$ of global sections of $(R, p, Q)$ has an orthogonality relation * given by $\sigma * t$ if for each $t \in Q$ either $\sigma(t)=0$ or $\tau(t)=0$ in $R / R_{t}$.

Let $R$ be a ring with an orthogonality relation * and an identity 1 .

For $Q^{\prime} \subseteq Q$ the map $I\left(Q^{\prime}\right): Q \rightarrow R$ is defined by

$$
I\left(Q^{\prime}\right)(t)= \begin{cases}\hat{I}(t) & \text { if } t \in Q^{\prime}, \\ 0 & \text { if } t \diamond Q^{\prime} .\end{cases}
$$

$I\left(Q^{\prime}\right)$ is called the indicator function of $Q^{\prime} \subseteq Q$, and $I\left(Q^{\prime}\right) \in \Gamma(R)$ if and only if $Q^{\prime} \subseteq Q$ is closed-open.

THEOREM 2.8. The mop $x+\hat{x}$ is a $x$-isomorphism of $R$ into $\Gamma(R)$. For each $\sigma \in \Gamma(R)$ there is a finite closed-open partition $\left(Q_{A_{i}}\right)$ of $Q$, and $x_{i} \in R$, such that $\sigma=\sum_{i} I\left(Q_{A_{i}}\right) \hat{x}_{i}$

Proof. For $x, y \in R, t \in Q$

$$
x-y(t)=\left(x-y+R_{t}, t\right)=\left(x+R_{t}, t\right)-\left(y+R_{t}, t\right)=\hat{x}(t)-\hat{y}(t),
$$

and similarly $\hat{x y}=\hat{x} \cdot \hat{y}$. If 1 is an identity in $R$ then for $\sigma \in \Gamma(R)$ and $t \in Q, \sigma \cdot \hat{I}(t)=\sigma(t) \cdot \hat{I}(t)=\sigma(t)$ so $\hat{i}$ is an identity in $\Gamma(R)$. If $\hat{x}(t)=0$ for each $t \in Q$ then $x^{* *} \in \prod_{t \in Q} t=(0)$ so that $x=0$.

If $x * y$ then $x^{* *} \cap y^{* *}=(0) \in \bigcap_{t \in Q} t$ so for each $t \in Q$ either 
$\hat{x}(t)=0$ or $\hat{y}(t)=0$, whilst if for each $t \in Q$ either $\hat{x}(t)=0$ or $\hat{y}(t)=0$ then $x^{* *} \cap y^{* *} \in \Pi t=(0)$; since * is regular this means that $x * y$.

Take $\sigma \in \Gamma(R)$. For each $t \in Q$ there is an $x_{t} \in R$ such that $\sigma(t)=\hat{x}_{t}(t)$. Then there is a closed-open neighbourhood $Q_{A_{t}}$ of $t$ such that $\sigma\left(t^{\prime}\right)=\mathfrak{x}_{t}\left(t^{\prime}\right)$ for all $t^{\prime} \in Q_{A_{t}}$. Then $\left\{Q_{A_{t}}: t \in Q\right\}$ is an open cover for $Q$ so there is a finite subcover $\left\{Q_{A_{t}}, \ldots, Q_{A_{t_{n}}}\right\}$. Put

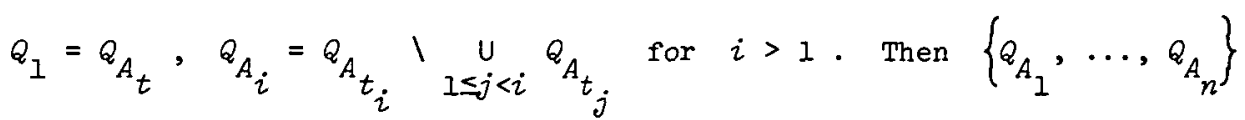
is a finite closed-open partition of $Q$. Put $x_{i}=x_{t_{i}}$, and consider the global section $\sum_{i} I\left(Q_{A_{i}}\right) \hat{x}_{i}:$ if $t \in Q_{A_{i}}$ then $\sum_{i} I\left(Q_{A_{i}}\right) \hat{x}_{i}(t)=\hat{x}_{i}(t)=\hat{x}_{t_{i}}(t)=\sigma(t)$. Thus, $\sigma=\sum_{i} I\left(Q_{A_{i}}\right) \hat{x}_{i} \cdot \quad / /$

DEFINITION 2.9. A ring $R$ with an orthogonality relation $*$ is said to be completely-projectable if for each $A \subseteq R, A^{*} \oplus A^{* *}=R$.

PROPOSITION 2.10. If $R$ is a ring with an orthogonality relation then the ring $\Gamma(R)$ of global sections of the sheaf $(R, p, Q)$ is completely-projectable.

Proof. For $Q^{\prime} \subseteq Q, x \in R$, define $I\left(Q^{\prime} ; x\right): Q \rightarrow R$ by

$$
I\left(Q^{\prime} ; x\right)(t)= \begin{cases}\hat{x}(t) & \text { for } t \in Q^{\prime}, \\ 0 & \text { for } t \gtrsim Q^{\prime} .\end{cases}
$$

Then $I\left(Q^{\prime} ; x\right) \in \Gamma(R)$ if and only if $Q^{\prime}$. is closed-open. As before, if $\sigma \in \Gamma(R)$ then $\sigma=\sum_{i} I\left(Q_{A_{i}} ; x_{i}\right)$ where $\left\{Q_{A_{i}}\right\}$ is a finite closed-open partition of $Q$. Now take $\left\{\sigma_{\alpha}\right\} \subseteq \Gamma(R)$ and $\sigma=\sum_{i} I\left(Q_{A_{i}} ; x_{i}\right) \in \Gamma(R)$. Put $S=\underset{\alpha}{\left.U \backslash t \in Q: \sigma_{\alpha}(t) \neq 0\right\}}$ and $S^{\prime}=Q \backslash S$. Then $S$ and $S^{\prime}$ are 
closed-open since $Q$ is extremally-disconnected. Thus

$\sigma=\sum_{i} I\left(Q_{A_{i}} n S ; x_{i}\right)+\sum_{i} I\left(Q_{A_{i}} n S^{\prime} ; x_{i}\right)$. Put $\sigma_{1}=\sum_{i} I\left(Q_{A_{i}} n S ; x_{i}\right)$, $\sigma_{2}=\sum_{i} I\left(Q_{A_{i}} n S^{\prime} ; x_{i}\right)$. Then $\sigma_{2} \in\left\{\sigma_{\alpha}\right\}^{*}$, for if $\sigma_{\alpha}(t) \neq 0$ for some $\alpha$, $t$ then $t \in S$ so that $\sigma_{2}(t)=0$. If $\tau \in\left\{\sigma_{\alpha}\right\}^{*}$, then $\sigma_{\alpha}(t) \neq 0$ for some $\alpha, t$ implies $\tau(t)=0$. That is, $\tau=0$ on $U\left\{t \in Q: \sigma_{\alpha}(t) \neq 0\right\}$, and since $\tau$ is continuous, $\tau=0$ on $S$. Then $\sigma_{1}=0$ on $S^{\prime}$ so that $\sigma_{1} * \tau$. That is, $\sigma_{1} \in\left\{\sigma_{\alpha}\right\} * *$ which means that $\left\{\sigma_{\alpha}\right\} * \oplus\left\{\sigma_{\alpha}\right\} * *=\Gamma(R)$

\section{Baer rings}

Let $R$ be a ring. For a subset $A \subseteq R$ the right annihilator of $A$ is the set $A^{0}=\{x \in R: a x=0$ for all $a \in A\}$. The left annihilator ${ }^{\circ} A$ is defined similarly. If $A=\{x\}$ is a singleton subset of $R$ then $A^{\circ},{ }^{\circ} A$ are denoted respectively by $x^{\circ},{ }^{\circ} x$.

DEFINITION 3.1. Let $R$ be a ring with identity $1 . R$ is said to be a complete Baer ring if for each $A \subseteq R$ there is an idempotent $e^{2}=e \in R$ such that $A^{\circ}=e R . R$ is said to be a Baer ring if for each $x \in R$ there is an idempotent $e$ with $x^{\circ}=e R$.

Thus in a complete Baer ring right annihilators of subsets are idempotently generated and similarly in a Baer ring right annihilators of elements are idempotently generated. A result of Kaplansky [3] shows that the assumption of an identity for the ring implies the same thing for left annihilators.

DEFINITION 3.2. A complete Baer ring is said to be of type $C$ if right annihilators of subsets are generated by central idempotents. A similar definition holds for Baer rings.

In a (complete) Baer ring $R$ the idempotent generator of a subset $A \subseteq R$ will be denoted by id $(A)$.

The set $B(R)$ of all central idempotents of $R$ is a complete boolean algebra for the operations 


$$
e \vee f=e f, e \wedge f=e+f-e f, e^{\prime}=1-e .
$$

Assume for the rest of this section that Baer ring means complete Baer ring.

PROPOSITION 3.3. If $R$ is a Baer ring then the relation $*$ on $R$ given by $x * y$ if $i d(x) \vee i d(y)=1$ is a regular orthogonality relation. Furthermore $e^{\rightarrow} e^{* *}$ is a complete isomorphism from $B(R)$ onto $B(R)$.

Proof. If $x * x$ then $i d(x)=1$ so that $x^{0}=R$ and thus $x=0$ since $l \in R$. To see that $x_{1} * y, x_{2} * y$ implies $\left(x_{1}-x_{2}\right) * y$ notice firstly that $\left(x_{1}-x_{2}\right)^{\circ} \supseteq x_{1}^{0} \cap x_{2}^{\circ}$. That is, $i d\left(x_{1}\right) R \cap i d\left(x_{2}\right) R \subseteq i d\left(x_{1}-x_{2}\right) R$ so that $\operatorname{id}\left(x_{1}\right) \wedge i d\left(x_{2}\right) \in i d\left(x_{1}-x_{2}\right) R$ and thus $\operatorname{id}\left(x_{1}\right) \wedge i d\left(x_{2}\right)=\operatorname{id}\left(x_{1}-x_{2}\right)\left[i d\left(x_{1}\right) \wedge i d\left(x_{2}\right)\right]$ which means that $\mathrm{id}\left(x_{1}-x_{2}\right) \geq \mathrm{id}\left(x_{1}\right) \wedge \mathrm{id}\left(x_{2}\right)$. Then $1 \geq i d\left(x_{1}-x_{2}\right) \vee i d(y) \geq\left[i d\left(x_{1}\right) \wedge i d\left(x_{2}\right)\right] \vee i d(y)=$ $\left[i d\left(x_{1}\right) \vee i d(y)\right] \wedge\left[i d\left(x_{2}\right) \vee i d(y)\right]=1$.

If $x * y$ and $z \in R$ then $(x z)^{\circ} \supseteq x^{\circ}$ so that $i d(x z) \geq i d(x)$, and thus $1 \geq i d(x z) \vee i d(y) \geq i d(x) \vee i d(y)=1$ so that $x z * y$. The remaining orthogonality properties of * follow easily.

To see that * is regular firstly notice that for $A \subseteq R$, $A^{*}=(1-i d(A))^{*}$ : if $e^{2}=e$ is an idempotent then $i d(e)=1-e$. Take $x \in A^{*}$, so that id $(x) \vee$ id $(a)=1$ for each $a \in A$ and thus

$$
\begin{aligned}
& \operatorname{id}(x) \vee \operatorname{id}(1-i d(1-i d(A)))=i d(x) \vee \operatorname{id}(A)= \\
& \qquad \quad \operatorname{dd}(x) \vee \bigwedge_{a \in A} \operatorname{id}(a)=\bigwedge_{a \in A} \operatorname{id}(x) \vee \operatorname{id}(a)=1 .
\end{aligned}
$$

Thus, $1-\operatorname{id}(A) \in A^{* *}$. If $y \in(1-i d(A)) *$ then for $a \in A$,

$$
\operatorname{id}(a) \vee i d(y) \geq i d(A) \vee i d(y)=i d(1-i d(A)) \vee i d(y)=1
$$

so that $y \in A^{*}$. Thus, $A^{*}=(1-\mathrm{id}(A))^{*}$.

Take $x, y \in R$. Then $x^{* *}=(1-i d(x)) * *, y^{* *}=(1-i d(y)) * *$ so that $[1-i d(x)] \cdot[1-i d(y)] \in x^{* *} \cap y^{* *}$. If $x * y^{*}$ then $i d(x) \vee i d(y) \neq 1$ so that $[1-i d(x)] \cdot[1-i d(y)] \neq 0$. That is, * is regular.

The map $e \mapsto e^{*}$ from $B(R)$ into $B(R)$ is surfective since 
$A^{*}=(1-i d(A))^{*}$. If $d^{*}=f^{*}$ then, since $1-e \in e^{*}, 1-f \in f^{*}$, it follows that $f-e f=e-f e$ so that $e=f$. That is $e \rightarrow e^{*}$ is injective. For a set $\left\{e_{\alpha}\right\} \subseteq B(R)$,

$$
\begin{aligned}
\left(\bigvee_{\alpha} e_{\alpha}\right)^{*} & =\left\{x \in R: \operatorname{id}(x) \vee \operatorname{id}\left(\bigvee_{\alpha} e_{\alpha}\right)=1\right\} \\
& =\left\{x \in R: \operatorname{id}(x) \vee\left(1-\bigvee_{\alpha} e_{\alpha}\right)=1\right\} \\
& =\left\{x \in R: \operatorname{id}(x) \vee \bigwedge_{\alpha}\left(1-e_{\alpha}\right)=1\right\} \\
& =\left\{x \in R: \bigwedge_{\alpha} \operatorname{id}(x) \vee\left(1-e_{\alpha}\right)=1\right\} \\
& =n_{\alpha} e_{\alpha}^{*} .
\end{aligned}
$$

Also $(1-e)^{*}=e^{* *}$, for if $x \in(1-e)^{*}, y \in e^{*}$ then

$$
\begin{aligned}
& \operatorname{id}(x) \vee \operatorname{id}(y)=[\operatorname{id}(x) \vee i d(y)] \wedge[e \vee(1-e)] \\
&=[\operatorname{id}(x) \vee e] \wedge[\operatorname{id}(x) \vee(1-e)] \wedge[\operatorname{id}(y) \vee e] \wedge[\operatorname{id}(y) \vee(1-e)] \\
&=[\operatorname{id}(x) \vee(1-e)] \wedge[\operatorname{id}(y) \vee e] \geq(1-e) \vee e=1,
\end{aligned}
$$

while on the other hand $1-e \in e^{*}$. Thus $e^{*} e^{* *}$ is a complete isomorphism onto $B(R)$, as asserted. //

PROPOSITION 3.4. If $R$ is a ring with identity $I$ and an orthogonality relation * then the ring $\Gamma(R)$ of global sections of the sheaf $(R, p, Q)$ is a complete Baer ring of type $C$ such that $\operatorname{id}(\sigma) \vee \operatorname{id}(\tau)=\hat{I}$ if and only if for each $t \in Q$ either $\sigma(t)=0$ or $\tau(t)=0$.

Proof. If $\sigma \in \Gamma(R)$ then $\sigma=\sum_{i} I\left(Q_{A_{i}}\right) x_{i}$, where $\left\{Q_{A_{i}}\right\}$ is a finite closed-open partition of $Q$. For a subset $\left\{\sigma_{\alpha}\right\}$ of $\Gamma(R)$ put $S\left(\sigma_{\alpha}\right)=\left\{t \in Q: \sigma_{\alpha}(t) \neq 0\right\}$ for all $\alpha$. Then $S(\sigma)=\bigcup_{i} Q_{A_{i}} \cap Q_{x_{i}^{*}}$ and is therefore closed-open so that $I(S(\sigma)) \in \Gamma(R)$. If $\left\{\sigma_{\alpha}\right\} \subseteq \Gamma(R)$ then the closure $\overline{U S\left(\sigma_{\alpha}\right)}$ of $U S\left(\sigma_{\alpha}\right)$ is closed-open, since $Q$ is extremally disconnected, so that $\left.I\left(\overline{U S\left(\sigma_{\alpha}\right.}\right)\right] \in \Gamma(R)$. If $\tau \in \Gamma(R)$ and $\sigma_{\alpha} \cdot \tau=0$ 
for all $\alpha$ then $\left\{t \in Q: I\left(\overline{U S\left(\sigma_{\alpha}\right)}\right)(t) \cdot \tau(t) \neq 0\right\}=\overline{U S\left(\sigma_{\alpha}\right)} \cap S(\tau)$ which is void so that $I\left(\overline{U S\left(\sigma_{\alpha}\right)}\right) \cdot \tau=0$. The converse is easily established, so that $\left\{\sigma_{\alpha}\right\}^{\circ}=\left[\hat{I}-I\left(\overline{U S S\left(\sigma_{\alpha}\right)}\right)\right] \cdot \Gamma(R)$ and $\hat{I}-I\left(\overline{U S S\left(\sigma_{\alpha}\right.}\right)$ is a centraI idempotent in $\Gamma(R)$. If $[\hat{I}-I(S(\sigma))] \vee[\hat{I}-I(S(\tau))]=\hat{I}$ then $I(S(\sigma)) \cdot I(S(\tau))=0$ so that for each $t \in Q$ either $\sigma(t)=0$ or $\tau(t)=0$. This argument reverses to show that if $\sigma \cdot \tau=0$ then $\operatorname{id}(\sigma) \vee \operatorname{id}(\tau)=\hat{i}$. $/ /$

PROPOSITION 3.5. Let $R$ be a complete Baer ring of type C. For the orthogonality relation * on $R$ given by $x * y$ if ia $(x) \vee$ ia $(y)=1$ the ring $R$ is *-isomorphic, $\phi: R \rightarrow \Gamma(R)$, to the Baer ring of global sections of the sheaf $(R, P, Q)$ and $\phi(\mathrm{id}(A))=\mathrm{id}(\phi(A))$ for each subset $A \subseteq R$.

Proof. The sections $\sigma \in \Gamma(R)$ can be written $\sum_{i} I\left(Q_{A_{i}^{*}}\right) \hat{x}_{i}$, where $x_{i} \in R$ and $\left\{Q_{A_{i}^{*}}\right\}$ is a finite closed-open partition of $Q$, so that $R \cong \Gamma(R)$ as a ring will follow once it is known that for each $A \subseteq R$, id(A) $=i d(\hat{A})$, where $i a(\hat{A})$ is the unique central idempotent in $\Gamma(R)$ satisfying $(\hat{A})^{\circ}=\operatorname{id}(\hat{A}) \cdot \Gamma(R)$. For $t \in Q$,

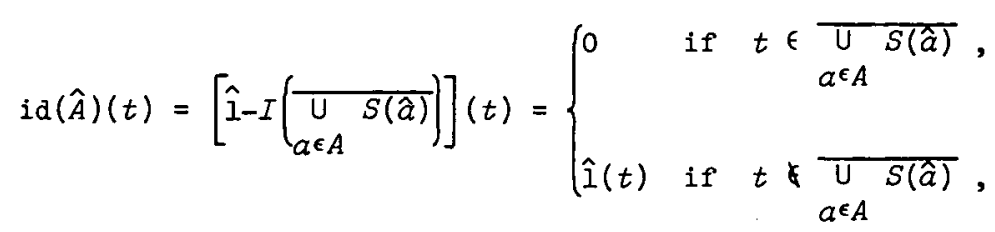

whilst $\operatorname{id}(A)(t)=\left(\operatorname{id}(A)+R_{t}, t\right)$, for $t \in Q$.

Take $t \in \overline{\bigcup_{a \in A} S(\hat{a})}$. Then $t=\underset{\alpha}{\lim }\left(t_{\alpha}\right)$, where $\left(t_{\alpha}\right)$ is a net in $\underset{\alpha \in A}{\cup} S(\hat{a})$. Then for each $\alpha$ there is an $a_{\alpha} \in A$ such that $\hat{a}_{\alpha}\left(t_{\alpha}\right) \neq 0$. Since $\operatorname{id}(A) \in(1-\mathrm{id}(A))^{*}=A^{*}$ then $R \supseteq A^{*} \vee \operatorname{id}(A)^{*} \supseteq A^{*} \vee A^{* *}=R$ which means that $A^{* *} \cap \operatorname{id}(A) * *=(0)$. Then, $\mathrm{id}(A)\left(t_{\alpha}\right)=0$ since 
$A^{* *} t_{\alpha}$ for each $\alpha$. Thus

$$
\operatorname{id}(A)(t)=\widehat{i d(A)}\left(\lim _{\alpha}\left(t_{\alpha}\right)\right)=\lim _{\alpha}\left(\widehat{i d(A)}\left(t_{\alpha}\right)\right)=0
$$

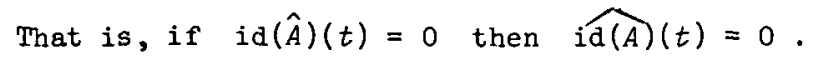

If $i \widehat{d(A)}(t) \neq 0$ then $\mathrm{id}(A)(t)=\hat{i}(t)$ since

$\operatorname{id}(A) * * \cap(1-i d(A)) * * \subseteq A^{*} \cap A^{* *}=(0)$ and thus $(1-\mathrm{id}(A)) * * \epsilon t$ since $t$ is prime. In this case $\operatorname{id}(\hat{A})(t)=\hat{1}(t)$ also: $A^{* *}=(1-i d(A)) * * t t$ so that $t \cup S(\hat{a})$ and thus $\operatorname{id}(\hat{A})(t)=\hat{\imath}(t)$. Thus the map $a \in A$

$\phi: R \rightarrow \Gamma(R)$ given by $\phi(x)=\hat{x}$ is a ring isomorphism onto $\Gamma(R)$.

Furthermore $\phi(\operatorname{id}(A))=\widehat{i d(A)}=i d(\hat{A})=i d(\phi(A))$. The map $\phi$ is a $*$-isomorphism since * is regular. //

\section{4. f-rings}

An $f$-ring is a lattice ordered ring $R$ for which $|x| \wedge|y|=0$ implies $|a x| \wedge|y|=0=|x a| \wedge|y|$ for each $a \in R$.

Thus, if * is the relation on $R$ given by $x * y$ if $|x| \wedge|y|=0$ then $*$ is an orthogonality relation. Furthermore $*$ is regular since $x^{* *} \cap y^{* *}=(|x| \wedge|y|) * *$.

The main result in $\$ 2$ gives the

PROPOSITION 4.1. Let $R$ be an f-ring with a unit. Then every ideal $R_{t}, \quad t \in Q$, is a prime lattice-ideal, $(R, p, Q)$ is a sheaf of totally ordered rings, and $\Gamma(R)$ can be lattice ordered so that $\Gamma(R)$ is an $f$-ring and $x \mapsto \hat{x}$ from $R$ into $\Gamma(R)$ is a lattice isomorphism into $\Gamma(R)$.

Proof. If $|y| \leq|x|, x \in R_{t}$ then $y^{* *} \subseteq x^{* *}$ so that $y \in R_{t}$. Thus $R_{t}$ is a lattice-ideal for $t \in Q$.

$$
\text { If }|x| \wedge|y|=0 \text { then } x^{* *} \cap y^{* *}=(0) \text { so that, for } t \in Q \text {, }
$$
either $x^{* *} \in t$ or $y^{* *} \in t$. That is, $x \in R_{t}$ or $y \in R_{t}$, so that $R_{t}, \quad t \in Q$, is a prime lattice-ideal. 
The factor spaces $R / R_{t}=\left\{\left(x+R{ }_{t}, t\right): x \in R\right\}$ can now be ordered by $\left(x+R_{t}, t\right) \geq 0$ if there is a $y \in R_{t}$ such that $x+y \geq 0$ in $R$. Then each factor space is a lattice-ordered ring and in fact, since each $R_{t}$ is a prime lattice ideal, the factor spaces are totally ordered. The ring $\Gamma(R)$ of global sections of the sheaf $(R, p, Q)$ can now be ordered pointwise: that is $\sigma \geq \tau$ if for each $t \in Q, \sigma(t) \geq \tau(t)$ in $R / R_{t}$. For $x \in R$ put $\operatorname{Pos}(x)=\{t \in Q: \hat{x}(t)>0\}$. Take $t_{0} \in \operatorname{Pos}(x)$ so that $\hat{x}\left(t_{0}\right)>0$ in $R / R_{t_{0}}$. By the definition of the order on $R / R_{t_{0}}$ there is a $y \in R_{t_{0}}$ such that $x+y>0$. Then $\hat{x}+\hat{y}\left(t_{0}\right)=\hat{x}\left(t_{0}\right)$ so that $\widehat{x+y}(t)=\hat{x}(t)$ for all $t \in Q_{A}$ where $Q_{A}$ is a neighbourhood of $t_{0}$. If $\widehat{x+y}$ assumes the value 0 in all neighbourhoods of $t_{0}$ then $\widehat{x+y}\left(t_{0}\right)=0$ contrary to the choice of $y$. Thus, there is a neighbourhood $Q_{B}$ of $t_{0}$ such that $\widehat{x+y}(t)>0$ for all $t \in Q_{B}$. Then $\hat{x}(t)=\widehat{x+y}(t)>0$ for all $t \in Q_{A} \cap Q_{B}$. That is, $Q_{A} \cap Q_{B} \subseteq \operatorname{Pos}(x)$ so that $\operatorname{Pos}(x)$ is open. On the other hand Pos $(x)$ is contained in the closed set $\{t \in Q: \hat{x}(t) \geq 0\}$, yet if $\hat{x}(t)=0$ then there is a neighbourhood $Q_{A}$ of $t$ such that $x\left(t^{\prime}\right)=0$ for all $t^{\prime} \in Q_{A}$ and thus $\operatorname{Pos}(x) \cap Q_{A}$ is void. Thus the closure $\overline{\operatorname{Pos}(x)}$ of Pos $(x)$ is contained in $\{t \in Q: \hat{x}(t) \geq 0\} \backslash\{t \in Q: \hat{x}(t)=0\}=\operatorname{Pos}(x)$ so that $\operatorname{Pos}(x)=\overline{\operatorname{Pos}(x)}$ is closed-open. Now for $x \in R$ the function $\hat{x} \vee 0: Q \rightarrow R$ given by $\hat{x} \vee O(t)=\max \{\hat{x}(t), 0\}$ in $R / R_{t}$ is just $I(\operatorname{Pos}(x)) \hat{x} \in \Gamma(R)$. Thus, if $\sigma=\sum_{i} I\left(Q_{A_{i}}\right) \hat{x}_{i} \in \Gamma(R)$ then for $t \in Q_{A_{i}}$, $\sigma \vee O(t)=\max \{\sigma(t), O\}=\max \left\{\hat{x}_{i}(t), 0\right\}$, so that with $Q$ $Q_{B_{i}}={ }_{A_{i}} \cap \operatorname{Pos}\left(x_{i}\right), \sigma \vee 0=\sum_{i}\left(Q_{B_{i}}\right) \hat{x}_{i} \in \Gamma(R)$. That is, $\Gamma(R)$ is lattice ordered. $\Gamma(R)$ is an $f$-ring for if $|\sigma| \wedge|\tau|=0$ then for each $t \in Q, \min \{|\sigma|(t),|\tau|(t)\}=0$ so that for $u \in \Gamma(R)$, 
$\min \{|\sigma u|(t),|\tau|(t)\}=0$ and thus $|\sigma u| \wedge|\tau|=0$.

\section{Commutative semi-prime rings}

In this section $R$ will denote a commutative semi-prime ring. There is a regular orthogonality relation * on $R$ given by $x * y$ if $x y=0$. The aim of this section is to prove the analogue for rings of a result of Spirason and Strzelecki [6] on vector lattices.

As before, $Q$ will denote the Stone space of the boolean algebra of polar subsets of $R,(R, p, Q)$ the sheaf of rings obtained from *, and $\Gamma(R)$ the ring of global sections of $(R, p, Q)$.

From $\$ 2$ it is known that for $t \in Q$ the ideal $R_{t} \subseteq R$ is prime. The next result gives a characterization of the ideals $R_{t}$ in the class of prime ideals of $R$ :

PROPOSITION 5.1. An ideal $I$ of $R$ is of the form $I=R_{t}$, for some $t \in Q$, if and only if

(1) I is a prime ideal;

(2) $x_{1}, \ldots, x_{m} \in I$ implies $x_{1}^{* *} \vee \ldots \vee x_{m}^{* *} \subseteq I$;

(3) $x \in I$ implies $x^{*} \neq(0)$.

Proof. If $x_{1}, \ldots, x_{m} \in R_{t}$ and $y \in x_{1}^{* *} \vee \ldots \vee x_{m}^{* *}$ then $y^{* *} \subseteq x_{1}^{* *} \vee \ldots \vee x_{m}^{* *} \in t$ (since $t$ is an ideal in $B(R)$ ) so that $y \in R_{t}$. If $x \in R_{t}$ then $x^{* *} \neq R$ since $x^{* *} \in t$ and $t$ is a proper ideal in $B(R)$. On the other hand let $I$ be an ideal in $G$ having the properties (1) - (3). Write $t_{0}$ for the ideal in $B(R)$ generated by the set $\left\{x^{* *} \vee y^{*}: x \in I, y \forall I\right\}$. That is,

$$
t_{0}=\left\{A^{*}: A^{*} \subseteq x_{1}^{* *} \vee \ldots \vee x_{m}^{* *} \vee y_{1}^{*} \vee \ldots \vee y_{n}^{*}: x_{i} \in I, y_{i} \vee I\right\} .
$$

If $t_{0}=B(R)$ then $R=(0)^{*}=x_{1}^{* *} \vee \ldots \vee x_{m}^{* *} \vee y_{1}^{*} \vee \ldots \vee y_{n}^{*}$ for some $x_{i} \in I, y_{i} I$. Then

$y_{1}^{* *} \cap \ldots \cap y_{n}^{* *}=\left(y_{1}^{*} \vee \ldots \vee y_{n}^{*}\right)^{*}=\left(y_{1}^{*} \vee \ldots \vee y_{n}^{*}\right)^{*} \cap\left(x_{1}^{* *} \vee \ldots \vee x_{m}^{* *}\right)$ 
so that $y_{1}^{* *} \cap \ldots \cap y_{n}^{* *} \subseteq x_{1}^{* *} \vee \ldots \vee x_{m}^{* *} \subseteq I$. Since each $y_{1}^{* *}$ is an ideal and $I$ is prime then $y_{i} \in I$ for some $i$, which is a contradiction so that $R t_{0}$. Thus $t_{0}$ is contained in a maximal ideal $t$. If $x \in I$ then $x^{* *} \in t_{0} \subseteq t$ so that $x \in R_{t}$. If $y \forall I$ then $y^{*} \in t_{0} \subseteq t$ so that $y^{* *} \forall t$ (since $y^{* *} \vee y^{*}=R \forall t$ ) and thus $y * R_{t}$. That is, $I=R_{t}$.

DEFINITION 5.2. A subset $I$ of $R$ is said to be a $*$-subset if $x_{1}, \ldots, x_{m} \in I$ implies $x_{1}^{* *} \vee \ldots \vee x_{m}^{* *} \subseteq I$. An element $x \in R$ for which $x^{*}=(0)$ is said to be a *-unit of $R$.

Thus the ideals $R_{t}, t \in Q$ are just the *-prime ideals not containing *-units.

PROPOSITION 5.3. If $M \subseteq R$ is a minimal prime ideal then $M=R_{t}$ for some $t \in Q$.

Proof. Take $x_{1}, \ldots, x_{m} \in M$ and suppose that for some $y \in x_{1}^{* *} \vee \ldots \vee x_{m}^{* *}, y^{* *} \Phi M$. Then $(0)=y^{*} \cap y^{* *}$ so that $y^{*} \subseteq M$. Thus $x_{1}^{*} \cap \ldots \cap x_{m}^{*} \subseteq y^{*} \subseteq M$, so that $x_{1}^{*} \subseteq M$ for some $i$.

Since $x_{i} \in M, M$ is prime and $R$ is semiprime, then there is an $a \gamma M$ such that $x a=0$. Thus $a \in x^{*}$ but $a \rtimes M$, which is a contradiction. Hence $x_{1}^{* *} \vee \ldots \vee x_{m}^{* *} \subseteq M$. If $x \in M$ then for some $a \star M, x a=0$ and thus $x^{\star} \neq(0)$.

The following result gives an internal description of those commutative semiprime rings for which the class $\left\{R_{t}: t \in Q, R_{t} \neq R\right\}$ is precisely the class of minimal prime ideals of $R$ :

DEFINITION 5.4. A commutative semiprime ring $R$ is said to be of locally compact type if for all $x, y \in R$ there exist $y_{1} \in x^{*}$, $y_{2} \in x^{* *}$ such that $y \in\left(y_{1}+y_{2}\right)^{* *}$.

PROPOSITION 5.5. A ring $R$ is of Zocally compact type if and only if each $R_{t} \neq R$ is minimal prime. 
Proof. Assume that $R$ is of locally compact type and $R_{t} \subseteq R$ is not minimal prime. Then for some $x \in R_{t}, x^{*} \subseteq R_{t}$. Now take $y \in R$. Then there exist $y_{1} \in x^{*}, y_{2} \in x^{* *}$ with $y \in\left(y_{1}+y_{2}\right)^{* *}$. Since $y_{1} * y_{2}$ then $y \in\left(y_{1}+y_{2}\right)^{* *}=y_{1}^{* *} \vee y_{2}^{* *} \subseteq R_{t}$ since $y_{1}, y_{2} \in R_{t}$. Thus $R_{t}=R$.

On the other hand if $R$ is not of locally compact type then there exist $x, y \in R$ such that $y \nless(x+a) * *$ for all $a \in x^{*}$. Put $S=\left\{(x+a)^{* *} \vee y^{*}: a \in x^{*}\right\}$. Then for $a \in x^{*}$, $\left[(x+a)^{* *} \vee y^{*}\right] \cap y^{* *}=(x+a)^{* *} \cap y^{* *} \neq y^{* *}$ since $y^{* *} \Phi(x+a)^{* *}$. Thus $(x+a) \vee y^{*} \neq R$ for each $a \in x^{*}$ so the ideal $t_{0} \subseteq B(R)$ generated by is a proper ideal and is therefore contained in a maximal ideal $t \subseteq B(R)$. Consider $R_{t}: y \in y^{* *} t$ since $y^{*} \in t$. Thus $y \notin R_{t}$ which means that $R_{t} \neq R$. Now suppose that $a^{* *} \cap x^{* *}=(0)$. Then $a * x$ since * is regular so that $a^{* *} \subseteq x^{* *} \vee a^{* *} \vee y^{*}=(x+a)^{* *} \vee y^{*} \epsilon t$ and thus $a \in R_{t}$. That is, $x \in R_{t}$ and $x^{*} \subseteq R_{t}$ so that $R_{t}$ is not minimal prime. $/ /$

\section{References}

[1] John Dauns and Karl Heinrich Hofmann, "The representation of biregular rings by sheaves", Math. 2. 91 (1966), 103-123.

[2] Orrin Frink, Jr, "Representations of Boolean algebras", Bull. Amer. Math. Soc. 47 (1941), 755-756.

[3] Irving Kaplansky, Rings of operators (Benjamin, New York, Amsterdam, 1968).

[4] Joseph Kist, "Compact spaces of minimal prime ideals", Math. 2. 111 (1969), 151-158.

[5] R.S. Pierce, "Modules over commutative regular rings", Mem. Amer. Math. Soc. 70 (1967).

[6] G. Spirason and E. Strzelecki, "A note on $P_{t}$-ideals" (submitted). 
[7] A.1. Veksler, "Linear spaces with disjoint elements and their conversion into vector lattices" (Russian), Leningrad. Gos. Ped. Inst. Učen. Zap. 328 (1967), 19-43.

Monash University,

Clayton, Victoria. 